\title{
The Stroop Effect Under the Interference of Language
}

$$
\text { Zongsheng Chen }{ }^{1,{ }^{*},{ }^{, \dagger} \text { Luyi Zou }{ }^{2, *},{ }^{*}, \dagger}
$$

\author{
${ }^{1}$ Wuhan Britain-China school \\ ${ }^{2}$ Dalian Ealing international school \\ *Corresponding author: ${ }^{*} 2917254167 @ q q . c o m,{ }^{b}$ guanghua.ren@gecacademy.cn \\ ${ }^{\dagger}$ These authors contribute equally.
}

\begin{abstract}
The Traditional Stroop effect proves that incongruent stimuli could delay people's reaction time, this is mainly because participants' attention was distracted by the interference factor. Based on this principle, our group reestablishes the Stroop effect, to find out the effect under the interference of language. This experiment is displayed in English_through Psychopy, and after the experiment, we found out that participants who had high proficiency in English spent a longer time naming the color of the contexts. Moreover, statistics showed that people with higher proficiency in English had a lower accuracy rate. This means that the proficiency of the language would negatively influence people during the Stroop test, perfectly reflect the basic Stroop effect.
\end{abstract}

Keywords: Language, Stroop effect, Time.

\section{INTRODUCTION}

In psychology, the Stroop effect is one of the bestknown phenomena. It has been reported by John Ridley Stroop in the published version of his dissertation in 1935. the basic Stroop task is to name the color in which a word is printed, ignoring the word itself. When the word is a color word printed in mismatched ink color, this is very difficult to do and results in slow, error-prone responding [1].

As in contemporary society, the Stroop effect has been widely used to solve some issues in reality. There are some typical examples.

\subsection{Application of the Stroop effect}

The Stroop effect can test one's identity, such as nationality and hobbies. Using the Stroop effect to test one's ability to do something could be very common in real life. A very typical example is a story recorded in the book 'Willpower', written by Baumeister \& Tierney [2]. During the cold war, the CIA highly suspected a person speaking fluent English was a Russian spy, but they couldn't find an effective way to prove his identity. Finally, the CIA let the spy did a simple Stroop test. During the tests, some words were written in Russian, and the name of the color was incongruent to the color of the inks. Not surprisingly, since the Russian spy could speak Russian, his reaction would be slower when reading those incongruent words, his identity was determined.

\subsection{Assumptions of the factors that might influence the Stroop effect}

From the examples mentioned above, our group noticed an important factor, i.e., language. The reason why the Russian spy gets caught was that he could speak Russia, so our group wanted to figure out whether language is an important factor of the Stroop effect, or the interference language had on the Stroop effect was just a coincidence. Other implements materials could help our group to make a valid assumption. Researchers from Cambridge had designed an experiment to discuss the Stroop effect in bilingualism. In their experiment, participants' age, age of acquisition of the second language, and proficiency in each language were analyzed. Participants consist of 71 Spanish-English bilinguals, 40 English monolinguals, and 11 Spanish monolinguals from South Florida. Proficiency in Spanish and English was established using a self-report questionnaire and the English and Spanish versions of the Boston Naming Test. In bilinguals, the Golden Stroop Test was administered in English and Spanish. Overall, performance was slower in bilinguals than in monolinguals. No significant differences were observed in color reading, but bilinguals performed worse in the naming color condition [3]. 


\subsection{Current research:}

It has been a long time since the Stroop effect is firstly proposed, some researchers hold a suspected view about the accuracy of the results of the Stroop test. For example, some researchers discover that when zero-dimensional correlation is created by random allocation of values, the Stroop effects vanished. These results imply that when the nominally irrelevant dimension is correlated with the relevant dimension, participants then attend to the irrelevant dimension and thus open themselves up to Stroop interference [4]. This research inspired our group to discuss the real relationship between our incongruent stimulus and to find out the appropriate variable to increase the validity of the results of our Stroop test. Under such circumstances, our group study about language's effect on the Stroop test, and decided to design a new experiment based on the basic Stroop effect. the independent variable of this research is language. For languages, our group chooses Chinese and English. Our group will use Psychopy to design our experiments. Our assumptions are based on an existing experiment. Compared to non-proficient bilinguals, proficient bilinguals showed better cognitive control at inhibiting irrelevant information, and they were slower at naming the color of the English words.[5]

\section{METHOD}

\subsection{Participant}

Our group tried to restrict the range of age of our participants because according to some research, age would influence the final results, for instance, As expected, the Stroop effect was greater for older than younger adults.[6]To reduce the effects of age, we recruited five volunteers from an international school for the experiment, two males and three females with the age from 16-22. The reason why participants are chosen within a limited range of age is to eliminate the influence caused by age as much as possible. Two of them have experience in English speaking country for more than three years, with more fluent English skill. The other three are the students from senior high school who always had lived in China, with the average English ability. To measure their English proficiency, each participants was given an English test. The content of the test is to translate the meaning of the words on the paper within a limited time of one minute. The number of correctly translated words was proportional to the participants' proficiency in English. The results were that participants who had lived abroad correctly 35 and 39 out of 100 words which are average difficulty. In comparison, a slightly less words translated correctly within a minute. Which is 33,33 , and 31 respectively. So as that participants who had lived abroad are more frequently in recognizing words.

\subsection{Design}

Stroop test is to let participants react the ink color of the words which within the congruent meaning of the ink [1].

What the difference with the traditional Stroop is that we paid more attention to the language used in the experiment. The words we show, which within the language of English. Setting up premise if the different English level does affect on reaction time. We separate people whether they have fluent English skills or not, to be the independent variable. By recording their reaction time to reflect their reaction condition, to be a dependent variable.

\subsection{Procure}

We represent the program of the experiment by Psychopy. [6] Which is software used to design Psychological experimental procedures. It is a platformindependent experimental control system written in the Python interpreted language using entirely free libraries. Before the experiment, we invite all the participants into a computer room, with five computers that run the procedure of the experiment. To avoid the influence of noise and other interruptions, we had ensured the room is quiet. Firstly, we explain the operations and rules to the participants and show them the introduction of the experiment. Then, all participants will start the experiment at the same time. To start the text, they first need to $\log$ in the information of their age, gender, and condition on the fluency of English, which is expressed by the years spent abroad. Or just sign in zero if they have lived in China. To start with, they will be shown the correct reaction on the keyboard when different colors of ink are seen. As well as the sign reads 'press the space to continue', which indicates they have already understood. Following the experiment start, whilst the time begins. They will be shown a range of words with the meaning of colors in congruent or incongruent colors of ink. For example, the correct press for yellow ink color is the 'up' button. Whatever pressing the correct button or not, the procedure will continue to the next word and the reaction time will be recorded by the procedure. During the experiment, every participant can do reaction to words they see. Though there was a long period provided for the advanced exercise, the randomly presented words still take different times for participants to react. It can be obverse that, someone with a higher language level evenly took a shorter time to do reaction than those with a lower language level. Furthermore, they all show the faster reaction as the meanings of words and the color of ink within the same. What's more, some individual factors seemed to take part in affecting reaction time. By the way, the time participants finish the experiments are different. 


\section{RESULTS}

These two tables show the data of our experiment. For participants who have high proficiency in English, their average reaction time is approximately 1.4 seconds, and their accuracy rate is $31 \%$. For participants who cannot speak English, their mean reaction time was 1.1 seconds, relatively shorter than the time used by bilingualism. What's more, their accuracy rate is $39 \%$, much bigger than proficient English speakers.

Table 1. The Mean reaction times

\begin{tabular}{ll}
\hline Group & Mean reaction time \\
\hline Proficient & 1.4079726 \\
Not proficient & 1.061344069 \\
\hline \multicolumn{1}{c}{ Table 2. The participants' mean accuracy rate } \\
\hline Group & Mean accuracy rate \\
\hline Proficient & $31 \%$ \\
Not proficient & $39 \%$ \\
\hline
\end{tabular}

\section{DISCUSSION}

\subsection{Discovery of our experiment:}

Through our experiment, our group could obviously discover the difference. People who could speak English had a longer response time than people who could not speak English, moreover, bilingual (Chinese and English) participants had greater probabilities to make a mistake than people who did not understand English.

\subsection{Compare and contrast}

According to the basic Stroop test, groups are divided into two of congruent and incongruent, however, in our experiment, the color of the text and the meaning of words are incongruent. Besides, we introduce a new variable: proficiency of a second language. Our experiments are displayed in English so our participants include people who could speak English and people who couldn't. For similarity, our group uses the same numerical indicators, reaction time, and rate of accuracy, to evaluate and explain our results.

\subsection{Explanation of the experiment's results}

The results were not surprising that people who speak English would spend a longer time reacting to the incongruent stimuli and are more likely to make mistakes. It might be because when people are responding to a specific stimulus, due to the interference of other factors, it is difficult for people to concentrate on responding to the stimulus.

\subsection{The discussion of the weaknesses of our test}

Our experiment still has several flaws:

Firstly, even though our experiment data has included the response time of people from a relatively wide range of ages, our data is still not diversified for us to determine or prove a common principle, because the total number of our experimental examples is not enough.

Secondly, our group assumed that our experiment might be influenced by other cognitive obstacles, such as color blindness. After introspection, we think we could find two brand-new stimuli to replace the color of the word and the ink color or choose participants with no color blindness.

Thirdly, the mechanism of our experiment might bring obstacles to our participants. Nearly half of the participants complained to us that they couldn't remember the instructions mentioned at the beginning during the exam, so it took them actual time to remind what key to type when they saw red, blue, or green. It was possible that they already figured out the correct answer, but they failed to type the correct key, which would influence their rate of accuracy. Our possible solution was before the test starts next time, we would first let the participants get familiar with the instructions, each of them is given the time of half an hour to be acquainted with the procedure by using the procedure themselves. Then making sure if they have understood by asking them some basic questions about specific operations.

Fourth, by viewing other successful examples, we found out that a lot of normative factors might contribute to inaccurate test results. For instance, research has developed a study about how sexual identity and degree of education could affect the Stroop effect.[8] Another experiments talked about how emotion would make a difference. These cases suggested us that we should better keep all our participants under same status.[9]

Fifth, considering synesthesia, which means the confusion of the nervous and sensory systems, and the simultaneous perception of multiple senses by one sensation.[10] For example, the Grapheme-color synesthesia, each word or number they see has a specific color. Hence the participants who has Grapheme-color synesthesia, will be affected by the color which comes to their minds as seeing the words. As well as doing effect on reaction time.[11]

\subsection{Future prospect of our research}

Our group hoped that the experiments we took could provide references to future development in the applications of the Stroop effect, such as:

First, in the Stroop effect, participants' reaction time could be a strong evidence in examining whether 
participants has been in early stage of Alzheimer or dementia [12];

Second, the joint research could also be very useful in clinical psychology, its main research and focus will be about social phobia, anxiety disorders, alcohol dependence, heroin, gambling, and compulsive disorders. [13]

\section{CONCLUSION}

This article reported a joint research of the basic Stroop test. And our group determined that the proficiency of the language could certainly influence the Stroop effect. It also revealed many new possible developments for the Stroop effect. We could assume more subjective factors that might bring influence on Stroop test besides our existing research.

\section{REFERENCES}

[1] Macleod, C. M. (2016). Stroop effect. Springer Berlin Heidelberg.

[2] Baumeister, F., \& Amp, R., Tierney, \& John. (2011).) Willpower: rediscovering our greatest strength. Penguin

[3] Rosselli M, Ardila A, Santisi MN, et al. (September 2002). "Stroop effect in Spanish-English bilinguals". Journal of the International Neuropsychological Society: JINS. 8 (6): 819 27. doi:10.1017/S1355617702860106. PMID 1224 0746

[4] Dishon-Berkovits, M., \& Algom, D. (2001). The stroop effect: it is not the robust phenomenon that you have thought it to be. Memory \& Cognition, 28(8), 1437-1449.

[5] Wang, R., Fan, X., Cong, L., \& Cai, Z. G. (2016). Cognitive control and word recognition speed influence the stroop effect in bilinguals. International Journal of Psychology, 51(2).

[6] West, R., \& Alain, C. (2010). Age-related decline in inhibitory control contributes to the increased stroop effect observed in older adults. Psychophysiology, 37(2), 179-189.

[7] Peirce, Jonathan W. (2007), PsychoPyPsychophysics software in Python [J/OL]. https://m.zhangqiaokeyan.com/openaccess_resources_thesis/0100037149041.html

[8] Elst, V. D., \& W. (2006). The stroop color-word test: influence of age, sex, and education; and normative data for a large sample across the adult age range. Assessment, 13(1), 62-79.
[9] Cheng, Z., \& Huang, Y. (2013). Emotional conflict effect in the face-word stroop task. Journal of Psychological Science, 36(4), 822-826.

[10] Megan S Steven, Colin Blakemore (2004). Visual synaesthesia in the blind. Perception. vol. 33, pp. 855-868. Visual Synaesthesia in the Blind

[11] Megan S Steven, Colin Blakemore (2004). Visual synaesthesia in the blind. Perception. vol. 33, pp. 855-868. Visual Synaesthesia in the Blind

[12] D H, Spieler, D A, Balota, M E, \& Faust. (1996). Stroop performance in healthy younger and older adults and in individuals with dementia of the alzheimer's type. Journal of experimental psychology. Human perception and performance.

[13] Chen, S. (2009). Researches and application of the emotional stroop effect in clinical psychology. Modern Applied Science, 2(1), 64. 